\title{
Sobre la relación entre Leptoglossus chilensis (Spinola, 1852) y Leptoglossus concaviusculus Berg, 1892 stat. rest. (Heteroptera: Coreidae), con nuevos datos acerca de su morfología
}

On the relationship between Leptoglossus chilensis (Spinola, 1852) and Leptoglossus concaviusculus Berg, 1892 stat. rest. (Heteroptera: Coreidae), with notes about their morphology

Eduardo I. Faúndez ${ }^{1,2} \&$ Mariom A. Carvajal ${ }^{1,2}$

\section{Resumen}

Se estudia la relación entre Leptoglossus chilensis y L. concaviusculus, se concluye que la segunda requiere rango de especie y no subespecie como se le ha tratado hasta ahora. Se entregan figuras de ambas especies incluyendo la genitalia masculina y se describe la ninfa $\mathrm{V}$ de L. concaviusculus.

\section{Palabras clave:}

Hemiptera, Coreinae, Anisosceleni, Leptoglossus, Sudamérica.

\begin{abstract}
The relationship between Leptoglossus chilensis and $L$. concaviusculus is studied. It is concluded that $L$. concaviusculus deserves full species rank. Figures of both species are included with emphasis on male genitalia. The $\mathrm{V}$ nymph of $L$. concaviusculus is described.
\end{abstract}

\section{Key words:}

Hemiptera, Coreinae, Anisoscelini, Leptoglossus, South America.

\section{INTRODUCCIÓN}

Coreidae es una numerosa familia de heterópteros que incluye unas 2550 especies clasificadas en cerca de 436 géneros (Faúndez, 2015). Los coreidos son principalmente fitófagos; sin embargo se les ha registrado consumiendo carroña $e$ incluso inflingiendo picaduras a seres humanos (Faúndez \& Carvajal, 2011). Muchas especies han sido consideradas de una alta importancia económica (Mitchell, 2000).

Leptoglossus Guérin-Méneville, 1831 es uno de los géneros mas diversos y complejos de la tribu Anisoscelini en el hemisferio occidental, incluyendo 61 especies con una de ella conformada por dos subespecies (Brailovsky, 2014). Las especies de este grupo son conocidas vernacularmente como chinches pata de hoja, por las dilataciones foliáceas presentes en sus metatibias. Este género se distribuye desde Canadá hasta la Patagonia (Faúndez \& Carvajal, 2011) y algunas especies han sido introducidas en el hemisferio oriental (Brailovsky, 2014). Este grupo fue revisado primeramente por Allen (1969), posteriormente Packauskas y Schaefer (2001) entregan nuevos datos y una clave para los grupos de especies; finalmente Brailovsky (2014) describe nuevos taxones, propone nuevas sinonimias y entrega una lista y clave actualizadas para el género.

Leptoglossus chilensis (Spinola, 1852) se ha señalado como una de las especies con más amplia distribución en este grupo, encontrándose desde Brasil hasta la Patagonia (Faúndez \& Carvajal, 2011). Ésta especie es generalista y ha sido reportada como causante

Entomology Department, School of Natural Resource Sciences, North Dakota State University, Fargo, ND, USA. $\triangle$ ed.faundez@gmail.com.

2 Departamento de Zoología Médica, Centro de Estudios en Biodiversidad (CEBCh), Magallanes 1979, Osorno, Chile. 
de daño en duraznos, ciruelas, uvas, higos y pomelos (Mitchell, 2000; González, 1989), siendo cuarentenaria para Estados Unidos de Norteamérica y Japón (González, 1989). Es la única especie de Leptoglossus que contiene subespecies: L. chilensis chilensis (Spinola, 1852) y L. chilensis concaviusculus Berg, 1892. La relación entre ambas permanece poco clara, siendo el objetivo de esta contribución estudiar el estatus de estas.

\section{MATERIALES Y MÉTODOS}

En terminología y morfología seguimos a Brailovsky (2014). Para el examen de genitalia los ejemplares han sido ablandados en solución de Barber y las estructuras han sido aclaradas en una solución saturada de $\mathrm{KOH}$, calentada a $100^{\circ} \mathrm{C}$ por una hora. Las fotografías fueron tomadas con una cámara digital adaptada a un microscopio estereoscópico. Las colecciones mencionadas en el texto son las siguientes: DARC: David A. Rider Collection, Fargo, USA; HRCC: Heteroptera Reference Collection CEBCh, Osorno, Chile. Todas las medidas se expresan en milímetros.

\section{RESULTADOS}

Allen (1969) propone tratar a $L$. concaviusculus como subespecie de L. chilensis basándose principalmente en la superposición de algunos caracteres externos lo que calificó como ejemplares intermedios, considerando que el estatus subespecífico con reservas era de momento la mejor opción. De este modo, L. chilensis chilensis quedaba distribuido en Chile y parte de Argentina, mientras que L. chilensis concaviusculus en Brasil y Argentina, siendo algunas provincias de este país el punto de superposición. Los autores posteriores no han profundizado en el tema y la situación de ambos taxones ha quedado pendiente, siendo la única especie de Leptoglossus en tener subespecies actualmente válidas. Cabe mencionar que Coscarón y Pall (2015) indican que Packauskas y Schaefer (2001) sinonimizan ambos taxones, sin embargo estos últimos autores especifican el rango subespecífico de $L$. concaviusculus dado por Allen (1969).

Las principales diferencias entre ambas subespecies son el tamaño de las dilataciones foliares de las tibias posteriores, manchas negras en el lóbulo posterior del pronoto; en adición con el exámen de nuevos ejemplares, hemos encontrado que la forma de la cabeza, ángulos humerales del pronoto y la genitalia masculina también varían entre $L$. chilensis chilensis y L chilensis concaviusculus, como se explica en detalle más adelante en las diagnosis.

De este modo nos parece que la evidencia es suficiente para considerar a ambos taxones con rango específico, con la correspondiente sinonimia asociada

Leptoglossus chilensis (Spinola, 1852)

(Figs. 1, 3, 5, 7)

(Anisoscelis chilensis Spinola)

= Theonoe spiniger Philippi, 1865 (Levi, 1962; basado en comunicación con W. E. China).

= Leptoglossus argentinus Bergroth, 1894 (Brailovsky, 2014)

\section{Diagnosis}

Cabeza compacta (Fig. 1), pronoto con manchas discoidales negras en el lóbulo posterior (Fig. 1), ángulos humerales del pronoto ligeramente compactados (Fig. 1), dilataciones foliares de las metatibias ligeramente ensanchadas, ocupando el $62 \%$ al $71 \%$ de la estructura (Fig. 3), pigóforo con aspecto ovoidal en vista ventral, con los lados levemente cóncavos hacia el ápice dándole un aspecto mas elongado (Fig. 5), parámeros con una sección convexa esclerotizada en el lado interno del lóbulo basal (Fig. 7).

Material examinado: ARGENTINA: Neuquén, 12-XII-1987, 1ðَ L. E. Peña leg. (HRCC), Río Negro, El Bolsón, 9-III-1982, 1 ㅇ L. E. Peña leg. (HRCC). CHILE: Región de Atacama, I-II-1992, 10 sin colector (HRCC); Región de Coquimbo, Pichidangui, XII-2012, 1 + Faúndez \& Carvajal leg. (HRCC); Región de Valparaíso, Placilla, III-2010, 3ðึ5우 L. Gutiérrez leg. (HRCC); Region de Valparaiso, Olmué, 7-IV-2013, 1ㅇ J. F Campodónico leg. (HRCC); Región Metropolitana: Cajón del 


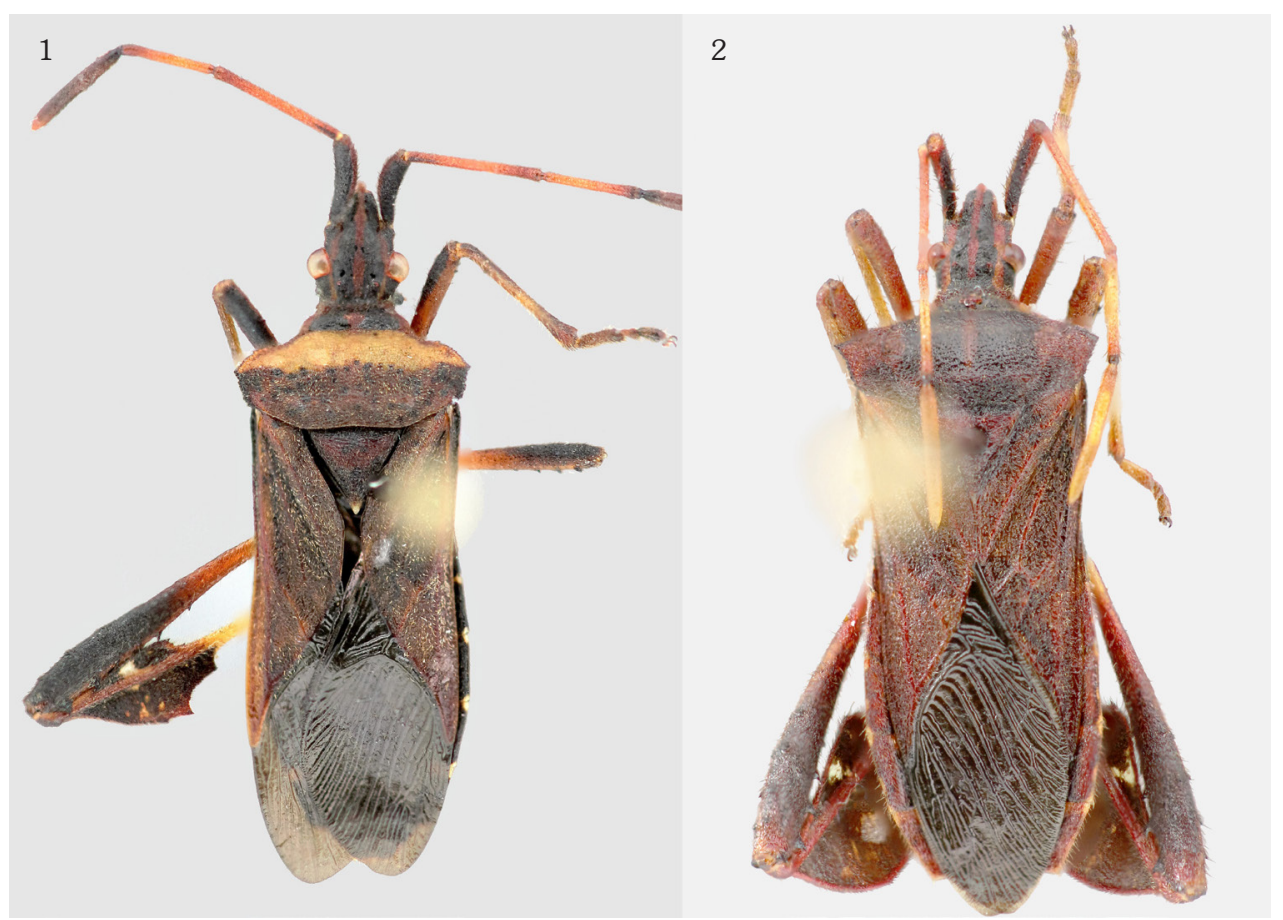

Fig.1. Leptoglossus chilensis, habitus; Fig. 2. Leptoglossus concaviusculus, habitus.

Maipo, 1§, R. Honour leg. (HRCC); Melipilla, 23-I-2009, 19 A. Lüer leg. (HRCC); Cajón del Maipo, 9-XI-1996, 1 đ̂ A. Lüer leg. (HRCC); Cajón del Maipo, Lagunillas, I-2009, 13 đ R. Pérez de Arce leg. (HRCC); Cajón del Maipo,

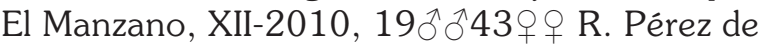
Arce leg. (HRCC); Cajón del Maipo, El Manzano,

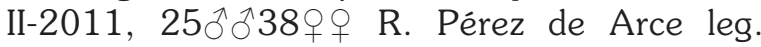
(HRCC); Cajón del Maipo, El Manzano, I-2013, 27ðð 72 우 R. Pérez de Arce leg. (HRCC);

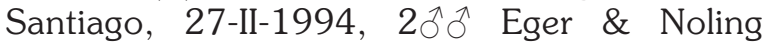
leg. (DARC), Región de O`Higgins, Rancagua, 3-II-2007, 2ðð 1 J. Aravena leg. (HRCC); Región del Maule, Vilches Alto, I-2010, 3 우 J. Villablanca leg. (HRCC); Región del Bío Bío, Periquillo, 2-XI-1994, 2ふふ7우 T. Cekalovic leg. (HRCC), Región de los Ríos, Panguipulli, 30-XII-2012, 1 đ3우으 J. F. Campodónico leg. (HRCC); Región de Los Lagos, Pto. Montt, XII1995, 2ðð T. Cekalovic leg. (HRCC).

Distribución (de acuerdo a Allen (1969) y esta contribución): Argentina (provincias de Catamarca, Córdoba, Mendoza, Neuquén, Río Negro, San Juan, y Salta), Chile (Región de Atacama a Región de Los Lagos). Cabe destacar que Artigas (1994) menciona esta especie presente desde la primera a undécima regiones de Chile; sin embargo esta distribución no se ha podido constatar y probablemente es errónea, como varias entregadas por Artigas (1994) para diversos heterópteros (Faúndez, 2016).

Leptoglossus concaviusculus Berg, 1892 stat. rest. (Figs. 2, 4, 6, 8, 9, 10, 11, 12, 13)

\section{Diagnosis}

Cabeza elongada (Fig. 2), pronoto sin manchas discoidales negras en el lóbulo posterior (Fig. 2), ángulos humerales del pronoto ensanchados (Fig. 2), dilataciones foliares de las metatibias ampliamente ensanchadas y alargadas, ocupando $71 \%$ al $82 \%$ de de la estructura (Fig. $4)$, pigóforo con aspecto ovoidal en vista ventral, con los lados fuertemente convexos (Fig. 6), parámeros rectos en la cara interna de la sección apical (Fig. 8).

Material examinado: ARGENTINA: Chaco,

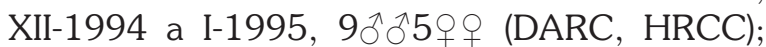


Chaco, Gral. Guemes, Pozo La Gringa, 11-I1996, 1ð1우 4 ninfas V (DARC, HRCC); Entre Ríos, Colón, III-1990, 2ðえ2우 Liebig leg. (DARC), Entre Ríos, Colón, IX-1988, 1 q Liebig leg. (DARC); Salta, $8.1 \mathrm{~km} \mathrm{~S}$ de Cafayate, 2-III2006, 1 3 우 D. A. Rider leg. (DARC, HRCC); Río Negro, General Conesa, III-2007, $1 q \mathrm{~J}$. Balbontín leg. (HRCC). BRASIL: Santa Catharina, Nova Teutonia, 1ð F. Plaumann leg. (HRCC); Sao Paulo, Cipó 12-I-1975, 1ð2우 V. N. Alin leg. (DARC). PARAGUAY: II-1972, 1 J. Hass (HRCC).

Distribución (de acuerdo a Allen (1969) y esta contribución): Argentina (provincias de Buenos Aires, Chaco, Córdoba, Corrientes, Entre Ríos, Formosa, Misiones, Neuquén, Río Negro, Salta, Santiago del Estero), Brasil (Santa Catharina, Sao Paulo, Rio Grande do Sul), Paraguay (Asunción), Uruguay (Montevideo).

Dentro del material examinado encontramos ninfas del último instar, las cuales no se conocen para esta especie, cuya descripción incluimos a continuación:

\section{Leptoglossus concaviusculus, ninfa V}

Cuerpo alargado (Fig.9), coloración general pardusca con toques negros rojizos y amarillentos.

Cabeza: Alargada, paraclipeos al nivel del clípeo, dos bandas negras atravesando transversalmente a nivel de los paraclípeos, ojos prominentes y ocelos situados a la altura de estos, dos pequeñas espinas rojizas delante de los ojos, antenas tetra-segmentadas, primer segmento claramente pasando el final de la cabeza, tubérculo antenífero inerme, búcula evanescente, rostro alcanzando las metacoxas, primer segmento rostral extendiéndose mas allá de los ojos anteriormente.

Tórax: Pronoto trapezoide, ángulos humerales producidos lateralmente (Fig.10), borde posterior dentado, margenes anterolaterales marfileños, cicatrices con dos procesos cónicos centrales rojos con aspecto espicular. Pterotecas elongadas alcanzando un tercio del abdomen y con dos procesos espinosos rojizos entre las bases de estas (Fig.11), protoescutelo levemente desarrollado. Patas parduscas, fémures metatorácicos con dos corridas de fuertes dientes
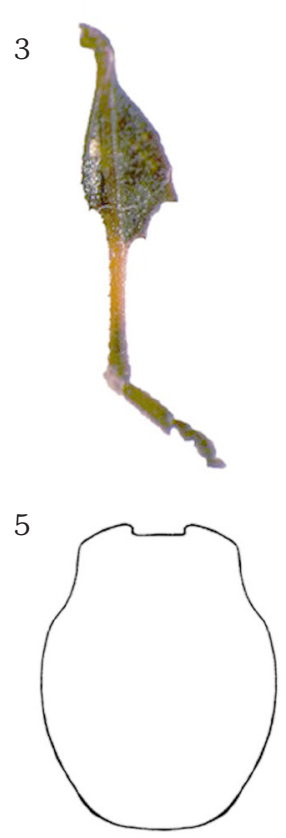

6
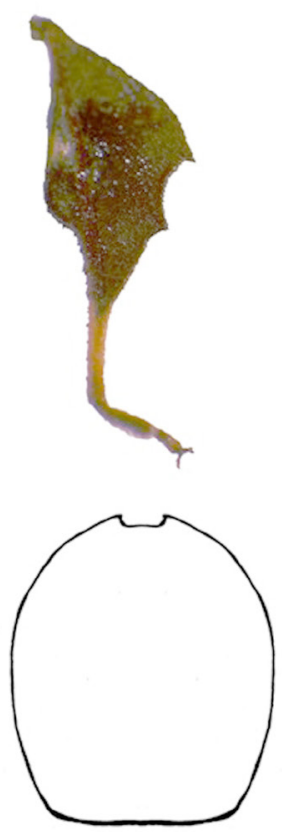

7

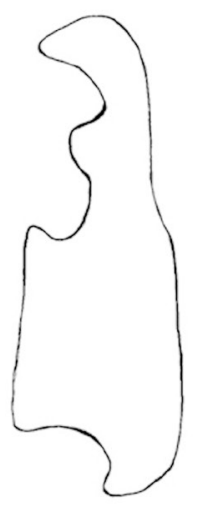

8

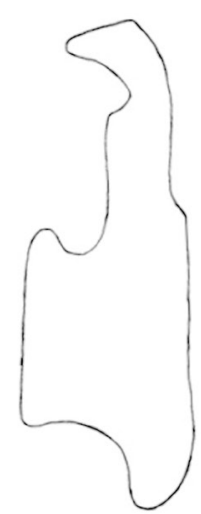

Figs. 3, 5, 7. Leptogossus chilensis, 3. Dilatación foliar de la metatibia, 5. Pigóforo, vista ventral, 7. Parámero. Figs. 4, 6, 8. Leptoglossus concaviusculus, 4. Dilatación foliar de la metatibia, 6. Pigóforo, vista ventral, 8. Parámero.

ventrales subapicales, tibia con sección foliácea ensanchada pero menos desarrollada que el adulto (Fig. 12).

Abdomen: Dos pares de glándulas dorsales (Fig. 13), presencia de un proceso con aspecto de espina, rojo por encima de cada abertura glandular, conexiva ondulada, sección ventral amarillenta, jaspeada de rojo. Segmentos abdominales terminales indefinidos, subcónicos.

Medidas ( $n=4)$ : Largo total: 7,91; ancho del pronoto: 3,01; largo del pronoto:1,60; largo de la 


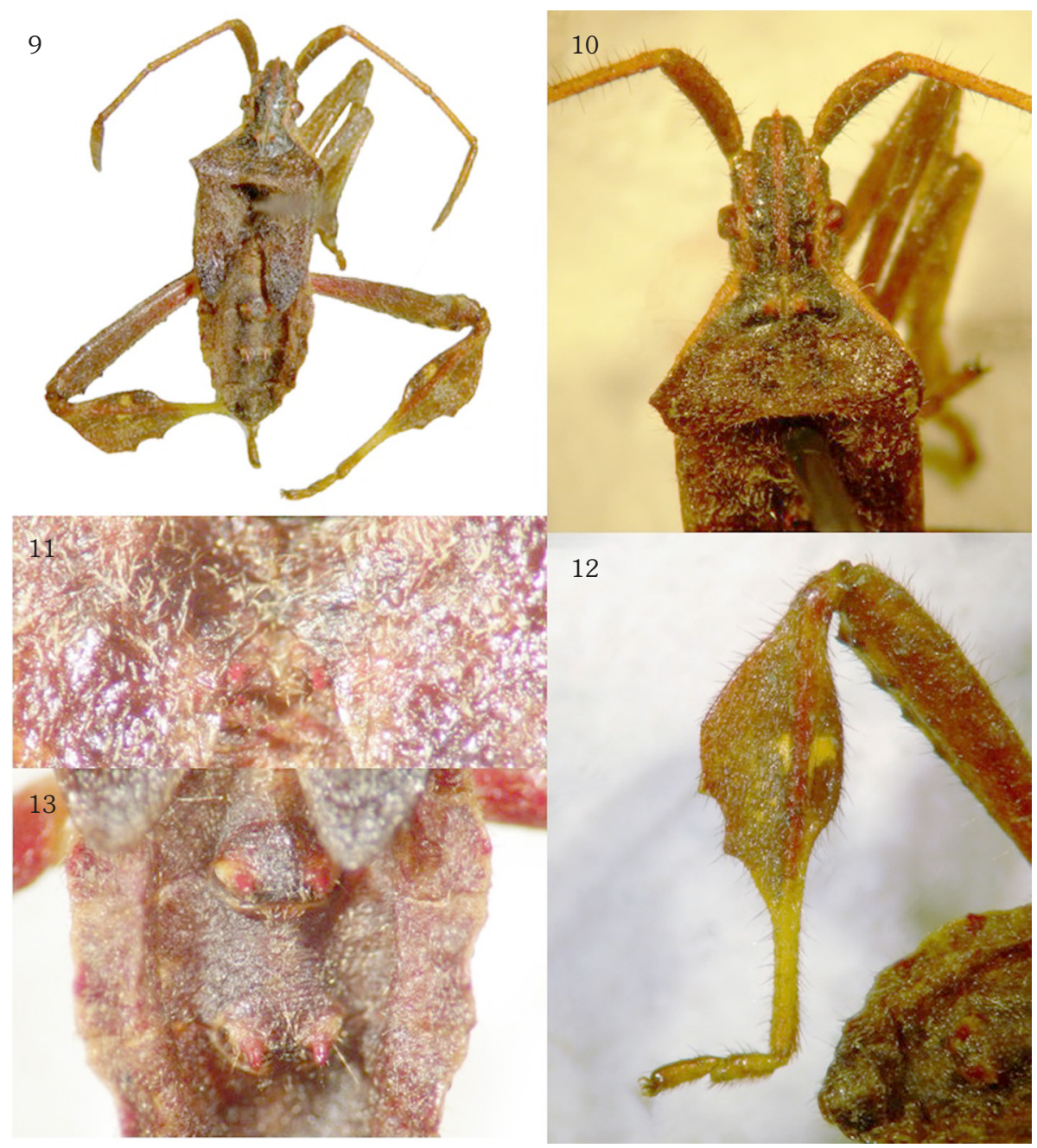

Figs. 9-13. Leptoglossus concaviusculus, Ninfa V, 9. Habitus, 10. Pronoto y cabeza, 11. Procesos entre las pterotecas, 12. Dilatación foliar de la metatibia, 13. Glándulas abdominales dorsales.

cabeza: 1,82; ancho de la cabeza: 1,50; largo de las pterotecas: 2,44 ; ancho de las pterotecas: 1,21 ; largo de la metatibia: 4,78; largo de la dilatación foliar de la metatibia: 3,14; ancho de la dilatación foliar de la metatibia: 1,48 ; antenómeros: $\mathrm{I}=1,35$ $\mathrm{II}=2,35 \mathrm{III}=1,47 \mathrm{IV}=2,15$; rostro: $\mathrm{I}=1,35 \mathrm{II}=1,26$ $\mathrm{III}=1,01 \mathrm{IV}=1,89$.

\section{DISCUSIÓN Y CONCLUSIONES}

Las diferencias presentes entre Leptoglossus chilensis y Leptoglossus concaviusculus son similares a las que pueden observarse entre diferentes especies del grupo de L. chilensis.
Adicionalmente detalles como la dilatación foliácea de la metatibia son un carácter de alta importancia a nivel especifico en este género (Packauskas \& Schaefer, 2001; Brailovsky, 2014), lo cual queda comprobado con el examen de la genitalia masculina, la que presenta buenas diferencias entre ambos taxones. Entre las largas series que hemos examinado, algunos ejemplares en el límite de la variabilidad pueden parecerse a una u otra especie, sin embargo los detalles de la genitalia son más constantes y se puede recurrir a ellos en caso de ejemplares dudosos. Debe, sin embargo, considerarse que ejemplares recientemente emergidos pueden tener estructuras genitales 
blandas; cuya forma puede ser confusa, la que si es inflada y suspendida en líquido, usualmente se puede apreciar su morfología sin mayores problemas. Es difícil establecer la relación entre las especies del grupo de L. chilensis, ya que todas son bastante cercanas y presentan un patrón de distribución andino, por lo que se requiere un análisis acabado del grupo para comprender su evolución y biogeografía. La ninfa V de L. concaviusculus se puede diferenciar de la de Leptoglossus impictus (Stål, 1859) (única conocida dentro de su grupo de especies) descrita por Diez et al. (2016), por la coloración general más oscura, el desarrollo mayor de los procesos espinosos rojizos en el cuerpo y la forma de la dilatación foliácea tibial (i.e. que es mucho mas gruesa) en $L$. concaviusculus.

Con los cambios aquí propuestos, el género Leptoglossus cuenta con 62 especies válidas.

\section{AGRADECIMIENTOS}

A Harry Brailovsky (UNAM) por atender nuestras permanentes consultas, a David A. Rider (NDSU), por permitirnos examinar el material depositado en su colección, a Gerald M. Fauske por las figuras 1 y 2 , a todos los colectores que han cedido su material para el desarrollo de este trabajo.

\section{LITERATURA CITADA}

Allen, R. C. (1969). A revision of the genus Leptoglossus Guérin (Hemiptera: Coreidae). Entomologica Americana, 45, 35-140.

Artigas, J. N. (1994). Entomología Económica. Concepción: Ediciones Universidad de Concepción, Chile.

Berg, C. (1892). Nova Hemiptera Argentinae et Uruguayensis. Anales de la Sociedad Científica Argentina, 33, 65-72.

Brailovsky, H. (2014). Illustrated key for identification of the species included in the genus Leptoglossus (Hemiptera: Heteroptera: Coreidae: Coreinae: Anisoscelini), and descriptions of five new species and new synonyms. Zootaxa, 3794(1), 143-178.
Coscarón, M. C., \& Pall, J. L. (2015). The tribe Anisoscelini (Hemiptera: Heteroptera: Coreidae) in Argentina. Zootaxa, 4033 (3), 411-426.

Diez, F., Ruiz Espindola, M., Cornelis, M., \& Coscarón, M. C. (2016). Additional information for Leptoglossus impictus (Stål, 1859) (Hemiptera: Heteroptera: Coreidae: Coreinae: Anisoscelini) from Patagonia, Argentina. Zootaxa, 4067(4), 494-500.

Faúndez, E. I. (2015). Synopsis of the genus Eldarca Signoret, 1864 (Hemiptera: Heteroptera: Coreidae). Zootaxa, 3915(2), 287-294.

Faúndez, E. I. (2016). On the isolated records of Triatoma infestans (Klug, 1834) (Heteroptera: Reduviidae: Triatominae) in Southern Chile. Arquivos Entomolóxicos, 15, 121-124.

Faúndez, E. I., \& Carvajal, M. A. (2011). Primer registro de una picadura de Leptoglossus chilensis (Spinola, 1852) (Hemiptera: Heteroptera: Coreidae) en un ser humano. Boletín de Biodiversidad de Chile, 6, 2225.

González, R. H. (1989). Insectos y Ácaros de importancia Agrícola y Cuarentenaria en Chile. Santiago de Chile: Editora Ograma.

Levi, H. W. (1962). Argyrodes Simon, Diponeura Simon, Robertus O. Pickard-Cambridge and Theonoe Simon (Arachnida: Araneae) proposed preservation under the plenary powers Z. N. (S.) 1481. Bulletin of Zoological Nomenclature, 19, 43-47.

Mitchell, P. L. (2000). Leaf-Footed Bugs (Coreidae). En C. W. Schaeffer \& A. R. Panizzi (Eds.), Heteroptera of Economic Importance (pp. 337-403). CRC Press, Boca Raton Fl.

Packauskas, R., \& Schaefer, C. W. (2001). Clarification of some taxonomic problems in Anisoscelini and Leptoscelini (Hemiptera: Coreidae: Coreinae). Proceedings of the Entomological Society of Washington, 103, 249-256

Spinola, M. (1852). Hemípteros. En C. Gay (Ed.), Historia física y política de Chile. Zoología, Vol. 7 (pp. 113-320), Paris. 